\title{
Impact of Imprecise Household Location on Effective Coverage Estimates Generated through Linking Household and Health Provider Data by Geographic Proximity
}

\section{Emily D Carter ( $D$ ecarter@jhu.edu )}

Johns Hopkins University Bloomberg School of Public Health https://orcid.org/0000-0003-1649-5274

\section{Melinda K Munos}

Johns Hopkins University Bloomberg School of Public Health

\section{Research}

Keywords: GIS, quality-adjusted coverage, linking, household survey, facility assessment, research methods

Posted Date: February 1st, 2021

DOl: https://doi.org/10.21203/rs.3.rs-156790/v1

License: (c) (i) This work is licensed under a Creative Commons Attribution 4.0 International License. Read Full License

Version of Record: A version of this preprint was published at International Journal of Health Geographics on August 21st, 2021. See the published version at https://doi.org/10.1186/s12942-021-00292-y. 


\section{Abstract}

Background: Combining household and health provider data can be used to estimate coverage of interventions and identify barriers to use. Without data on specific sources of care utilized by individuals, researchers often assign individuals to healthcare providers based on geographic proximity. The Demographic and Health Survey (DHS), a common source of population health data, does not collect data on the location of participant households. They present displaced data on the central point within household clusters. This may introduce error into analyses based on the distance between households and providers.

Methods: We assessed the effect of imprecise household location on quality-adjusted effective coverage of child curative services estimated by linking sick children to providers based on geographic proximity. We used data on care-seeking for child illness and health provider quality in Southern Province, Zambia. The dataset included the location of respondent households, a census of providers, and data on the exact outlets utilized by sick children included in the study. We displaced the central point of each household cluster point five times. We calculated quality-adjusted coverage by assigning each sick child to a provider's care based on three measures of geographic proximity (absolute distance, travel time, and geographic radius) from the household location, cluster point, and displaced cluster locations. We compared the estimates of quality-adjusted coverage to each other and estimates calculated using each sick child's true source of care.

Results: Fewer children were linked to their true source of care using cluster locations than household locations. Estimates of coverage were not statistically different using different measures of geographic proximity or household location. Estimates did not vary significantly from estimates produced using each sick child's true source of care.

Conclusions: Use of original or displaced cluster location did not produce statistically different coverage estimates than using household location. However, it did reduce the proportion of children that linked to their true source of care. The limited effect of household location imprecision on quality-adjusted coverage estimates could be due to a lack of variability in provider quality. These findings may not hold in a setting with more considerable variation in provider quality. This work was supported by the Bill \& Melinda Gates Foundation, Grant Number INV-006966

\section{Background}

Combining data from household and health facility assessments can be used to assess barriers to intervention coverage and estimate effective coverage of essential health service settings (1) and assess barriers to improved population health. Data from household surveys provide a population-based denominator of intervention need and care-seeking for services, such as the proportion of pregnant women who delivered in a health facility. Health provider assessments offer information on provider quality, including available infrastructure, commodities, equipment, human resources, and potentially 
provision of care. Linking these two data sources can provide a more complete picture of the care likely received by a population, for example, the proportion of women who delivered with a health care provider with sufficient structural resources and competence to provide appropriate labor and delivery care. Linking household data on intervention need and care-seeking with data on provider quality can produce not only informative effective coverage estimates, assessing the proportion of individuals in need of an intervention who received quality services, but also actionable data to differentiate between utilization and quality bottlenecks in achieving desired health outcomes.

Various methods for linking household and provider data exist (2). Exact-match linking, or assigning individuals to their specific source(s) of care, is the most accurate method of linking. However, ecological linking methods, such as assigning an individual to one or more providers based on geographic proximity or administrative catchment area, are often more feasible because they do not require data on the specific source of care. These methods are feasible for use with household surveys such as the Demographic and Health Survey (DHS) and Multiple Indicator Cluster Survey (MICS), which ask about the type of provider utilized but not the specific name of the provider or facility. A review by Do and colleagues found most analyses have linked households or individuals to healthcare providers based on geographic proximity, rather than the reported source of care, making assumptions about service access and use (2). Do noted most analyses also used existing data sets, predominantly DHS household data and Service Provision Assessment (SPA) facility data (2). Existing household surveys typically collect imprecise household location data, potentially introducing bias into analyses based on geographic proximity. DHS collect data on a single central population point within a sampling cluster, or enumeration area, rather than the location of individual households. For confidentiality reasons, the DHS randomly displaces the location of the cluster central point up to $5 \mathrm{~km}$ (1\% up to $10 \mathrm{~km}$ ) in rural areas and $2 \mathrm{~km}$ in urban areas to preserve respondent confidentiality (3). MICS does not collect GIS data on a regular basis and refer data users to contact country statistics offices to access cluster locations mapped in census cartography (4).

Guidelines on the use of DHS GPS data note that use of displaced DHS location data can increase the bias and error for analyses using the distance between clusters and resources as a covariate (5). An analysis by Skiles and colleagues found moderate, random misclassification of distance to a health provider and service environment when measured using displaced cluster locations (6). This analysis assessed two types of ecological linking approaches ( $5 \mathrm{~km}$ buffer and administrative unit) and did not have a measure of true source of care utilized by the subjects.

In a previous analysis, we assessed the amount of bias introduced to estimates of effective coverage of child curative services generated using different ecological linking methods against estimates generated using a gold-standard exact-match linking method for a population in Southern Province, Zambia (7). We found most ecological linking methods produced statistically equivalent estimates when conditioning the ecological linking on type of provider from which care was sought for the illness. However, those ecological linking analyses which employed measures of geographic proximity used data on the exact location of each sick child's primary residence. 
Using data on care-seeking for child illness and health provider quality in Southern Province, Zambia, we assessed the potential error introduced to effective coverage linking analyses by using original and displaced cluster central point location in place of household location. We assessed the proportion of children linked to their true source of care using original and displaced cluster locations and compared estimates of quality-adjusted coverage of curative child health services generated using measures of geographic proximity based on household location, undisplaced cluster location, and displaced cluster location to gauge bias in estimates.

\section{Methods}

\section{Study design, data collection, and key measures}

We performed a secondary analysis of data collected in Southern Province, Zambia as part of a study assessing the feasibility and performance of exact-match and various ecological linking methods. A detailed description of the study methods and findings has been published previously (7). Briefly, we conducted the study in five health facility catchment areas in Choma district between January and March 2016. The study collected data on care-seeking for illness in children under 5 (fever, diarrhea, or suspected ARI) in the preceding two weeks, using a household survey instrument based on the Zambia DHS. In addition to the standard DHS questions on the type of provider from which care was sought for reported child illness, we also asked mothers to identify (name or describe) the specific source(s) of care utilized. We also collected data on structural quality, or infrastructure required, for managing child illness for every health care provider in the study area using questions derived from the Service Availability and Readiness Assessment (SARA). The structural quality indicators were designed to assess a provider or facility's capacity to provide curative services for children, including the presence of drugs and commodities, training, supervision, and provider case management knowledge. We included public, private, informal, and traditional sources of care in the assessment. Geo-locations of all participating households and health care providers were collected using the geopoint function built into Open Data Kit (ODK) Collect operated on Motorola Moto G (Gen 2) smartphones running the Android 5.0.2 system.

\section{Analysis}

\section{Overall approach}

We used data generated using the exact-match approach as the measure of correct quality-adjusted effective coverage of management of child illness in the study population. Using the exact-match linking approach, we assigned each child the structural quality score of the specific provider from which care was reportedly sought, which was considered to be the true source(s) of care. Children were not linked using the exact-match method if their caregiver could not recall the name of the provider or facility from 
which care was sought or the provider could not be located for inclusion in the study, mostly affecting individuals who utilized informal shops.

To simulate ecological linking in the absence of data on specific source of care, each sick child was linked to the closest health provider(s) within the reported category of source of care (Box 1 ) using three measures of geographic proximity: 1) absolute distance, 2) travel time, and 3) $5 \mathrm{~km}$ radius. Each measure of geographic proximity was applied using 1) known household location, 2) undisplaced cluster location, and 3) five sets of displaced cluster locations, each reported separately. Quality-adjusted coverage of management of child illness was calculated using each combination of ecological linking method and measure of sick child location by assigning each child the quality score of the proximal provider(s) to which they were linked.

\begin{tabular}{|l|}
\hline Box 1. Categories of healthcare providers in the study area \\
\hline Public \\
\hline Government hospital \\
\hline Government health center / post \\
\hline Government CBA / fieldworker \\
\hline Private \\
\hline Private hospital / clinic \\
\hline Pharmacy \\
\hline Informal \\
\hline Shop / market \\
\hline Traditional / faith-based practitioner \\
\hline
\end{tabular}

For both the exact-match and each ecological linking approach, we calculated the quality-adjusted coverage of management of child illness as the average quality score across all sick children in the study. If no care was sought for a sick child, they were assigned a quality score of zero. If care was sought from multiple sources, we averaged the scores of those sources.

To quantify the bias introduced into each method by imprecise household location, we compared the estimates of quality-adjusted coverage from each combination of ecological linking approach and cluster location against the 1) exact-match quality-adjusted coverage estimates and 2) estimates generated using each ecological approach with the true household location. We also assessed how accurately each 
approach identified the actual provider(s) utilized by each sick child by comparing the provider(s) linked to each sick child using the ecological approaches with the specific source(s) of care reported by each child's mother.

\section{Quality score}

A full description of the construction of provider structural quality scores and methods for defining geographic proximity is presented in a previous publication (7). Briefly, we defined each provider's structural quality score as the availability of services, commodities, and human resources needed to appropriately manage common child illnesses (Box 2). These indicators were considered the minimum inputs for appropriate care: the basic commodities required to diagnose and treat common child illness, along with the human resources and clinical knowledge to apply them correctly. As such, the score reflects an upper ceiling of the potential quality of care offered by a provider. We calculated scores as a continuous variable ranging from zero (no capacity to provide care) to $100 \%$ (full capacity to provide care). 


\section{Box 2. Structural quality score components}

\section{Diagnostics}

Malaria Diagnostic (RDTs or microscopy)

Malnutrition Diagnostic (MUAC or Scale + Height board + Growth chart)

ARI Diagnostic (Stethoscope or respiratory timer)

General microscopy (Functioning microscope and slides)

\section{Basic Medicines}

ORS

Zinc

ACT

Oral antibiotic

\section{Severe / Complicated Illness Medicines}

IV fluids

Injectable quinine or artesunate

Injectable antibiotics

\section{Human Resources}

Training (At least one staff member with IMCl or relevant training)

Guidelines (IMCl guidelines or relevant guidelines or job aid available)

Supervision (Received supervision visit with case management observation in past 3 months)

\section{Available Services}

Diagnosis and treat malaria (by pathology)

Diagnosis and treat diarrhea (by pathology)

Diagnosis and treat ARI (by pathology) 
Diagnosis and treat malnutrition (by pathology)

Facilitated referral capacity

Knowledge

Average performance on case scenarios

\section{Geographic proximity}

We employed three measures of geographic proximity in this analysis. For each method, we developed an automated script in QGIS comparable to the process outlined for application in ArcGIS in a previous paper (7). We conducted all geographic analyses in QGIS 2.18.24 (Open Source Geospatial Foundation Project, Beaverton, OR, USA). Ecological linking was restricted to only assign children to the types of providers (managing authority and level of care) from which care was reportedly sought based on responses during the household survey. For example, if a mother she reported care for her sick child from a government health center, then the child could only be linked to another government health center - not a private facility or a government hospital.

- Absolute distance: Each sick child was linked to the single closest provider based on absolute distance from the child's location within the reported source of care provider category. This method is the simplest approach for assigning a child to a specific provider.

- Travel Time: Each sick child was linked to the single closest provider by travel time from the child's location within the reported source of care provider category. Travel time was approximated by grading the relative speed of travel on different types of roads (e.g. paved roads, graded roads, footpaths). This method is designed to model the effect of road access and quality on care-seeking.

- $5 \mathrm{~km}$ Radius: Each sick child was linked to all providers within the source of care provider category within a $5 \mathrm{~km}$ radius of the child's location. This method is designed to approximate a 1-hour walking distance from a household to a provider in any direction.

\section{Cluster location and displacement}

The central point location for each cluster was generated to capture an area of high population density within each cluster inline the DHS central point measurement procedures. A census of all households within each of the study catchment areas was conducted before the study and included the location of each household. In QGIS, we grouped all the households into clusters of 150 households based on measured latitude and longitude, and we calculated the mean point of each cluster of 150 households as the central point. 
Each central point was displaced five times using an R script developed by Measure Evaluation for DHS cluster displacement (5). In brief, the code offsets each point using a random angle and random distance, capped at $5 \mathrm{~km}$ for rural clusters ( $1 \%$ capped at $10 \mathrm{~km}$ ) and $2 \mathrm{~km}$ for urban clusters. The code further restricts the displacement to ensure points are not displaced outside of their true administrative unit (e.g., district). However, this feature was redundant in our analysis due to the small size of the study area. We ran the displacement code in R 3.4.3 (R Foundation for Statistical Computing, Vienna, Austria) and we imported each set of displaced coordinates into QGIS for the linking analyses.

We then substituted each central point and displaced central point for the household location in our measures of geographic proximity. Instead of calculating the geographic proximity of providers from the home of each sick child, we measured proximity from the relevant central point or displaced central point location as depicted in Figure 1.

\section{Results}

A full description of the study population, loss to follow-up, and healthcare provider characteristics are available in a previous publication (7).

Among the 1084 children included in the household care-seeking survey, $35 \%$ of urban children and $36 \%$ of rural children experienced at least one illness meeting DHS criteria in the two weeks preceding the survey, primarily fever (Table 1). Most mothers (79\% rural; 67\% urban) reported seeking care for their child's illness. Most children sought care from a skilled provider, including government health facilities, government community-based agents (CBAs), and private clinics. Government health centers were the primary reported source of care in both the urban $(60 \%)$ and rural $(61 \%)$ areas. In the rural area, $18 \%$ of children were taken to a CBA for care. In the urban area, care was sought for $5 \%$ of children from informal shops. Hospitals, pharmacies, private facilities, and traditional practitioners accounted for a small number of care-seeking events. 
Table 1. Characteristics of reported child illness and care-seeking events, by stratum

\begin{tabular}{|c|c|c|c|c|c|c|}
\hline & \multicolumn{3}{|c|}{ Rural } & \multicolumn{3}{|c|}{ Urban } \\
\hline & $\mathrm{n}$ & $\%$ & $\mathrm{Cl}$ & $\mathrm{n}$ & $\%$ & $\mathrm{Cl}$ \\
\hline & 547 & & & 537 & & \\
\hline Proportion of children with at least one DHS illness & 199 & 36.4 & $\begin{array}{l}{[32.4-} \\
40.5]\end{array}$ & 186 & 34.6 & $\begin{array}{l}\text { [30.7- } \\
38.8]\end{array}$ \\
\hline Reported Child IIIness & 199 & & & 186 & & \\
\hline Diarrhea & 23 & 11.6 & $\begin{array}{l}{[7.8-} \\
16.8]\end{array}$ & 50 & 26.9 & $\begin{array}{l}{[21.0-} \\
33.7]\end{array}$ \\
\hline Fever & 117 & 58.8 & $\begin{array}{l}{[51.8-} \\
65.4]\end{array}$ & 85 & 45.7 & $\begin{array}{l}{[38.7-} \\
52.9]\end{array}$ \\
\hline$A R I^{1}$ & 6 & 3 & $\begin{array}{l}{[1.4-} \\
6.6]\end{array}$ & 3 & 1.6 & $\begin{array}{l}{[0.5-} \\
4.9]\end{array}$ \\
\hline Diarrhea \& Fever & 28 & 14.1 & $\begin{array}{l}{[9.9-} \\
19.6]\end{array}$ & 35 & 18.8 & $\begin{array}{l}{[13.8-} \\
25.1]\end{array}$ \\
\hline Diarrhea \& ARI & 3 & 1.5 & $\begin{array}{l}{[0.5-} \\
4.6]\end{array}$ & 0 & 0 & - \\
\hline Fever \& ARI & 17 & 8.5 & $\begin{array}{l}{[5.4-} \\
13.3]\end{array}$ & 10 & 5.4 & $\begin{array}{l}{[2.9-} \\
9.7]\end{array}$ \\
\hline Diarrhea, Fever, \& ARI & 5 & 2.5 & $\begin{array}{l}{[1.0-} \\
5.9]\end{array}$ & 3 & 1.6 & $\begin{array}{l}{[0.5-} \\
4.9]\end{array}$ \\
\hline
\end{tabular}

Proportion of illnesses for which mother reported seeking care from:

Any provider

Skilled provider ${ }^{2}$

$>1$ provider
199

$\begin{array}{llllll}157 & 78.9 & \begin{array}{l}72.7- \\ 84.0]\end{array} & 124 & 66.7 & {[59.6-} \\ & & & & 73.1]\end{array}$

151

$116 \quad 62.4 \quad[55.2-$
186

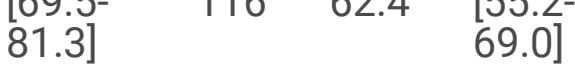

$\begin{array}{llllll}9 & 4.5 & {[2.4-} & 5 & 2.7 & {[1.1-} \\ & & 8.5] & & & 6.3]\end{array}$

Proportion of children that sought care from category of provider:

Govt hospital
199

186

$0 \quad 0$

5

2.7

[0.9-

6.2] 


\begin{tabular}{|c|c|c|c|c|c|c|}
\hline Govt health center / post & 122 & 61.3 & $\begin{array}{l}{[54.2-} \\
68.1]\end{array}$ & 111 & 59.7 & $\begin{array}{l}{[52.3-} \\
66.8]\end{array}$ \\
\hline Govt CBA / fieldworker & 36 & 18.1 & $\begin{array}{l}{[13.0-} \\
24.2]\end{array}$ & 1 & 0.5 & $\begin{array}{l}{[0.0-} \\
3.0]\end{array}$ \\
\hline Pvt hospital / clinic & 0 & 0 & - & 1 & 0.5 & $\begin{array}{l}{[0.0-} \\
3.0]\end{array}$ \\
\hline Pharmacy & 1 & 0.5 & $\begin{array}{l}{[0.0-} \\
2.8]\end{array}$ & 2 & 1.1 & $\begin{array}{l}{[0.1-} \\
3.8]\end{array}$ \\
\hline Shop / market & 2 & 1 & $\begin{array}{l}{[0.1-} \\
3.6]\end{array}$ & 9 & 4.8 & $\begin{array}{l}{[2.2-} \\
9.0]\end{array}$ \\
\hline Traditional / faith-based practitioner & 5 & 2.5 & $\begin{array}{l}{[0.8-} \\
5.8]\end{array}$ & 0 & 0 & - \\
\hline \multicolumn{7}{|c|}{${ }^{1}$ ARI defined as cough with chest-related difficulty breathing } \\
\hline \multicolumn{7}{|c|}{${ }^{2}$ Skilled providers included government and private health facilities and government CBAs } \\
\hline
\end{tabular}

Most skilled providers offered moderate to high levels of structural quality for managing child illnesses. Figure 2 presents structural quality scores by provider categories. Structural quality scores varied most by category of provider, and in most cases did not vary greatly within provider categories used in the geographic linking. While there were a few providers whose scores were notably above or below others within their category, these were provider categories that were uncommon sources of care such as pharmacies and traditional practitioners. A detailed description of scores by provider type is available in a previous publication (7).

We were able to link most children to their exact reported source of care, including $99 \%$ of rural careseeking events and $93 \%$ of urban care-seeking events (Table 2). In the rural areas, we observed a greater distance in the shift from household location to central point location due to the low density of households in these areas requiring a greater geographic catchment to generate clusters of 150 households (Figure 3). Using the household location, cluster central point, and displaced central point locations, we were able to link all children to a provider within the reported category of care using both the absolute distance and travel time methods as neither method capped the maximum distance to link to a provider. All urban children were linked to a provider within the reported care category using the $5 \mathrm{~km}$ radius method, however only $63.8 \%, 81.3 \%$, and 47.6 to $72.9 \%$ of rural children linked to any provider using household, cluster central point, or displaced cluster location, respectively.

\section{Table 2. Percent children linked to any provider within care category by method and strata}




\begin{tabular}{|lll|}
\hline & Rural & Urban \\
\hline Exact-match & $\%$ & $\%$ \\
\hline Absolute Distance & 98.8 & 93 \\
\hline HH Location & 100 & 100 \\
\hline EA Central point & 100 & 100 \\
\hline EA Displaced A & 100 & 100 \\
\hline EA Displaced B & 100 & 100 \\
\hline EA Displaced C & 100 & 100 \\
\hline EA Displaced D & 100 & 100 \\
\hline EA Displaced E & 100 & 100 \\
\hline Travel Time & & \\
\hline HH Location & 100 & 100 \\
\hline EA Central point & 100 & 100 \\
\hline EA Displaced A & 100 & 100 \\
\hline EA Displaced B & 100 & 100 \\
\hline EA Displaced C & 100 & 100 \\
\hline EA Displaced D & 100 & 100 \\
\hline EA Displaced E & 100 & 100 \\
\hline 5 km Radius Buffer & & \\
\hline HH Location & 63.8 & 100 \\
\hline EA Central point & 81.3 & 100 \\
\hline EA Displaced A & 59.0 & 100 \\
\hline EA Displaced B & 72.9 & 100 \\
\hline EA Displaced C & 47.6 & 100 \\
\hline EA Displaced D & 70.5 & 100 \\
\hline EA Displaced E & 69.3 & 100 \\
\hline
\end{tabular}

Using household location, $89 \%$ of rural and $88.3 \%$ of urban children linked to their true reported source of care using absolute distance (Table 3). A lower proportion (73.2\% rural; $84.5 \%$ urban) of children were 
linked to their true source of care when cluster central point location was used in place of household location. The proportion linked to their true source when using the displaced central point location ranged from 66.3 to $74.1 \%$ among rural children and 72.9 to $79.2 \%$ among urban children.

\section{Table 3. Percent children linked to true source of care by method and strata}

\begin{tabular}{|lll|}
\hline & Rural & Urban \\
\hline & $\%$ & $\%$ \\
\hline Absolute Distance & & \\
\hline HH Location & 89 & 88.3 \\
\hline EA Central point & 73.2 & 84.5 \\
\hline EA Displaced A & 74.1 & 72.9 \\
\hline EA Displaced B & 66.3 & 74.4 \\
\hline EA Displaced C & 71.7 & 76 \\
\hline EA Displaced D & 67.5 & 76.7 \\
\hline EA Displaced E & 72.9 & 79.1 \\
\hline Travel Time & & \\
\hline HH Location & 78 & 76.7 \\
\hline EA Central point & 56.1 & 45.8 \\
\hline EA Displaced A & 57.2 & 35.7 \\
\hline EA Displaced B & 64.5 & 46.5 \\
\hline EA Displaced C & 41 & 45.7 \\
\hline EA Displaced D & 54.8 & 35.7 \\
\hline EA Displaced E & 71.7 & 27.9 \\
\hline
\end{tabular}

Compared to absolute distance, a lower proportion (78\% rural; $76.7 \%$ urban) of children linked to their true reported source of care using travel time from household location (Table 3). The proportion linked to their true source of care fell to $56.1 \%$ of rural and $45.8 \%$ of urban when using cluster central point location. The proportion linked to their true source when using the displaced central point location ranged from 41 to $64.5 \%$ among rural children and 27.9 to $46.5 \%$ among urban children.

Despite the low to moderate proportion of children who linked to their true source of care using cluster central point and displaced cluster locations, all geolinking methods produced similar quality-adjusted coverage estimates compared to the precise exact-match method which assigned children to their true 
source of care (Figure 4). Differences in quality-adjusted coverage estimated using the absolute and $5 \mathrm{~km}$ radius geolinking methods with varying underlying location data were minor and inconsistently under and overestimated the exact-match effective coverage estimates. In both the rural and urban strata, the travel time approach produced consistently lower quality-adjusted coverage point estimates across locations, but they were not statistically different from the exact-match or other geolinked estimates. None of the estimates generated using the central point location or displaced central point location were statistically different from the estimates generated using the specific household location.

\section{Discussion}

We found that ecological methods based on geographic proximity accurately linked children to their true source(s) of care in most cases in this rural sub-Saharan African setting. However, using cluster central point location as a proxy for household location increased the proportion of children assigned to incorrect sources of care. Displacement of those central points variably increased and decreased the proportion linked to their true source of care. Despite this limited accuracy in identifying the true source(s) of care, estimates of quality-adjusted coverage of management of child illness generated using the ecological linking methods and central point locations did not differ significantly from estimates generated using data on the true source of care.

The primary reason for the lack of effect on quality-adjusted coverage estimates may have been the limited variation in structural quality within key categories of providers. Both DHS and MICS ask mothers to report on the type of provider from which care was sought for a child illness (e.g., govt health facility, private clinic, traditional practitioner, etc). This is also true for other questions around service contact and care-seeking, such as place of delivery and source of postnatal care. We, therefore, used the type of provider from which care was sought to restrict the linked provider options to only providers within the reported source of care category. This restriction meant that although children may not have been linked to their exact source of care, they were linked to a provider of the same level and managing authority. Within those provider categories that were most commonly utilized by the study population, namely government health facilities and CBAs, the structural quality was reasonably consistent. As a result, a child could be linked to any provider within those categories and would experience a similar level of structural quality.

However, we do not expect these results to hold in settings where quality within provider categories is more variable. This analysis was limited by its setting, which was characterized by relatively homogenous provider quality and care-seeking behavior, so we cannot generalize our findings to settings where care-seeking patterns are more diverse and provider quality is less consistent. Further, our measure of provider quality focused on structural factors and provider knowledge. It did not include gold-standard assessments of provider quality based on direct observation of care with clinical reassessment, which might have produced a more variable measure of provider quality.

\section{Conclusions}


This analysis suggests that use of displaced cluster central point location data in ecological linking analyses does not significantly bias measures of quality-adjusted coverage in settings where providers within the same general geographic area and provider category supply broadly consistent quality of care. However, it does provide evidence that using cluster central point or displaced data can introduce error when defining specific sources of care based on geographic proximity, even in settings where most children utilized the closest provider. Caution should be used when interpreting measures of geographic proximity generated using non-specific location data such as cluster central points and displaced points.

\section{Abbreviations}

ARI - Acute respiratory infection

CBA - Community-based agent

DHS - Demographic and Health Survey

GIS - Geographic information system

GPS - Global Positioning System

MICS - Multiple Indicator Cluster Survey

SARA - Service Availability and Readiness Assessment

SPA - Service Provision Assessment

\section{Declarations}

\section{Ethics approval and consent to participate}

Ethical approval for the study that produced the data used in this secondary analysis was obtained from the Institutional Review Boards of Johns Hopkins School of Public Health and Excellence in Research Ethics and Science (ERES) Converge in Zambia.

\section{Consent for publication}

Not applicable

\section{Availability of data and materials}

The datasets analyzed during the current study are available from the corresponding author on reasonable request. Household GIS data cannot be shared due to participant confidentiality concerns.

\section{Competing interests}


The authors declare that they have no competing interests

\section{Funding}

This work was supported by the Bill and Melinda Gates Foundation [grant number OPP1172551].

\section{Authors' contributions}

EC and MM conceptualized the study. EC conducted the analysis and drafted the manuscript. All authors read and approved the final manuscript.

\section{Acknowledgements}

Not applicable

\section{References}

1. Amouzou A, Leslie HH, Ram M, Fox M, Jiwani S, Requejo J, et al. Advances in the measurement of coverage for RMNCH and nutrition: from contact to effective coverage. BMJ Global Health. 2019;e001297.

2. Do M, Micah A, Brondi L, Campbell H, Marchant T, Eisele T, et al. Linking household and facility data for better coverage measures in reproductive, maternal, newborn, and child health care: systematic review. J Glob Health. 2016 Dec;6(2):020501.

3. Burgert CR, Colston J, Roy T, Zachary B. Geographic displacement procedure and georeferenced data release policy for the Demographic and Health Surveys. 2013 [cited 2018 Sep 14]; Available from: https://dhsprogram.com/publications/publication-SAR7-Spatial-Analysis-Reports.cfm

4. FAQ - UNICEF MICS [Internet]. [cited 2019 Jun 19]. Available from: http://mics.unicef.org/faq

5. Perez-Haydrich C, Warren JL, Burgert CR, Emch ME. Guidelines on the use of DHS GPS data. 2013 [cited 2018 Sep 14]; Available from: https://dhsprogram.com/publications/publication-SAR8-SpatialAnalysis-Reports.cfm

6. Skiles MP, Burgert CR, Curtis SL, Spencer J. Geographically linking population and facility surveys: methodological considerations. Popul Health Metr. 2013 Aug 8;11(1):14.

7. Carter ED, Ndhlovu M, Eisele TP, Nkhama E, Katz J, Munos M. Evaluation of methods for linking household and health care provider data to estimate effective coverage of management of child illness: results of a pilot study in Southern Province, Zambia. J Glob Health. 2018 Jun;8(1):010607.

\section{Figures}




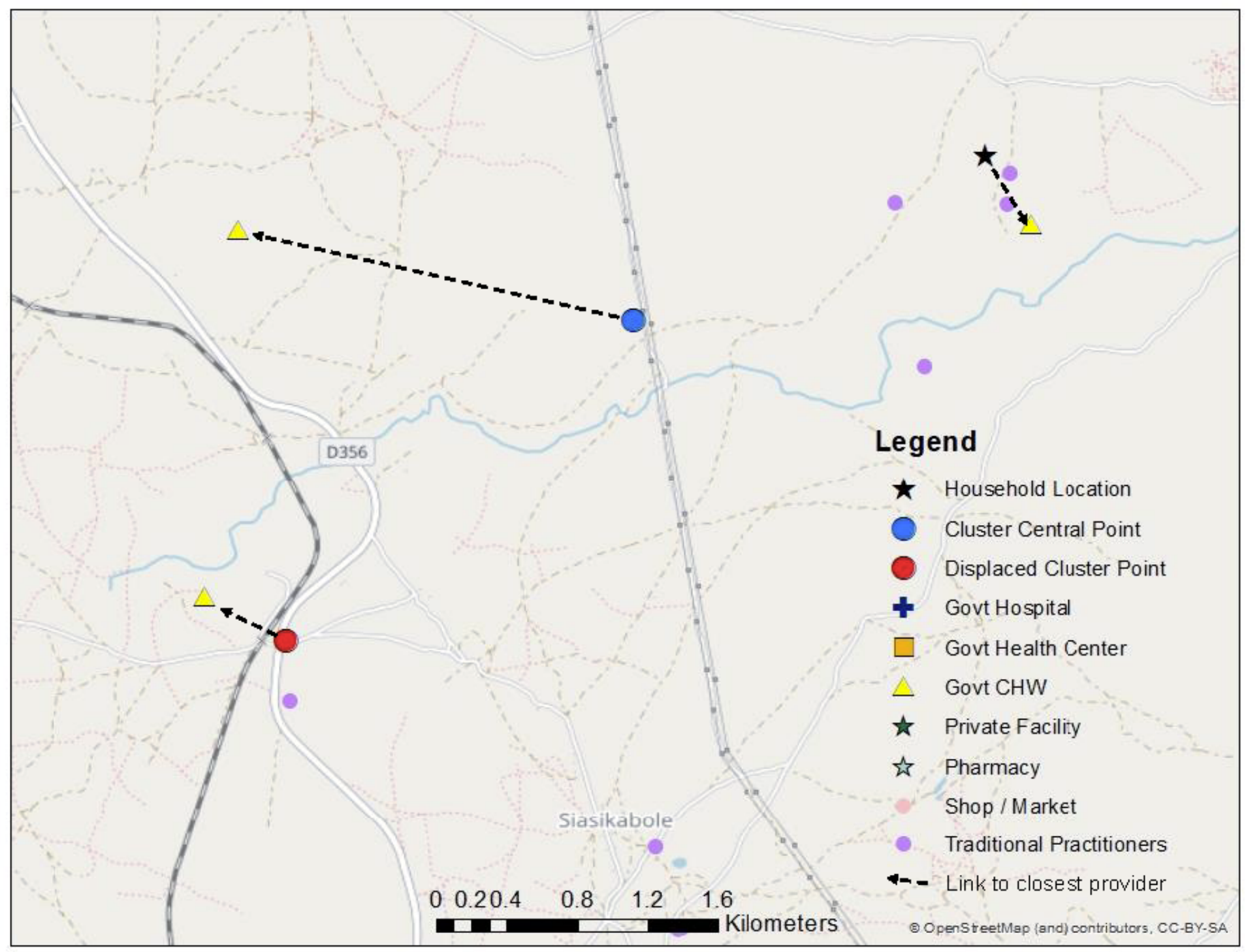

Figure 1

Map of link to closest $\mathrm{CHW}$ based on true household location, cluster central point, and displaced cluster point 


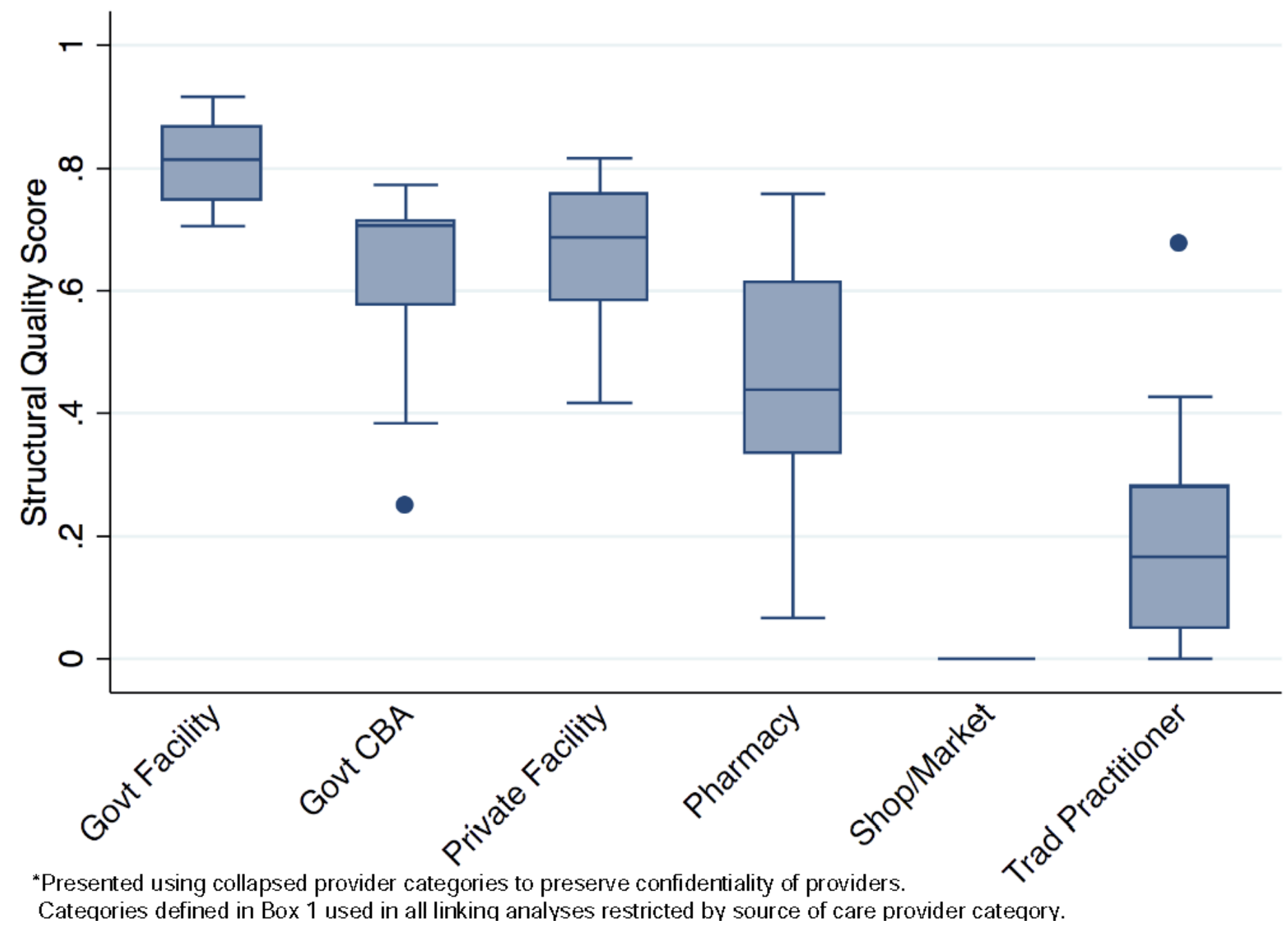

Figure 2

Median and IQR of structural quality scores by provider category 


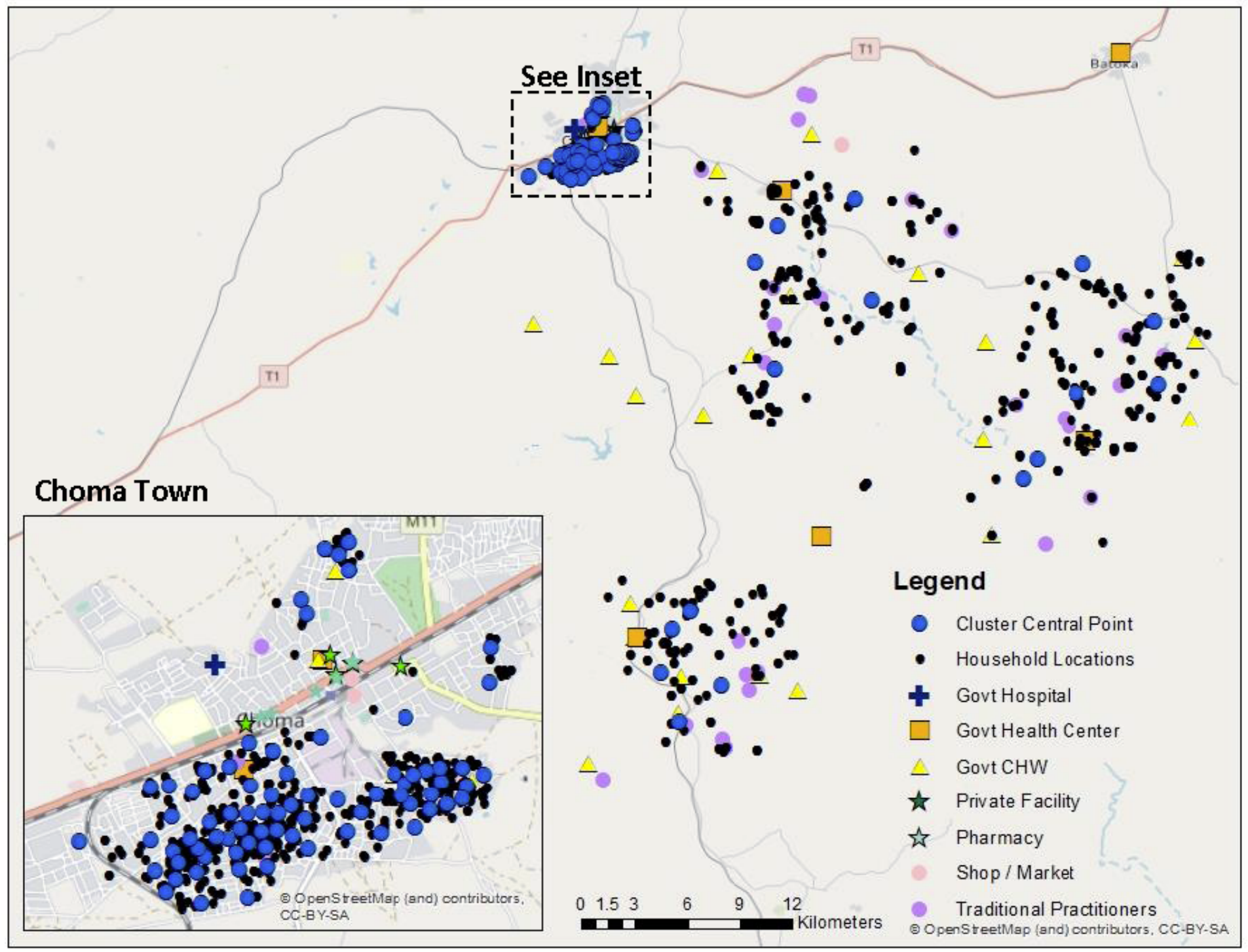

Figure 3

Map of cluster central points, households, and providers (update to include household census points) 


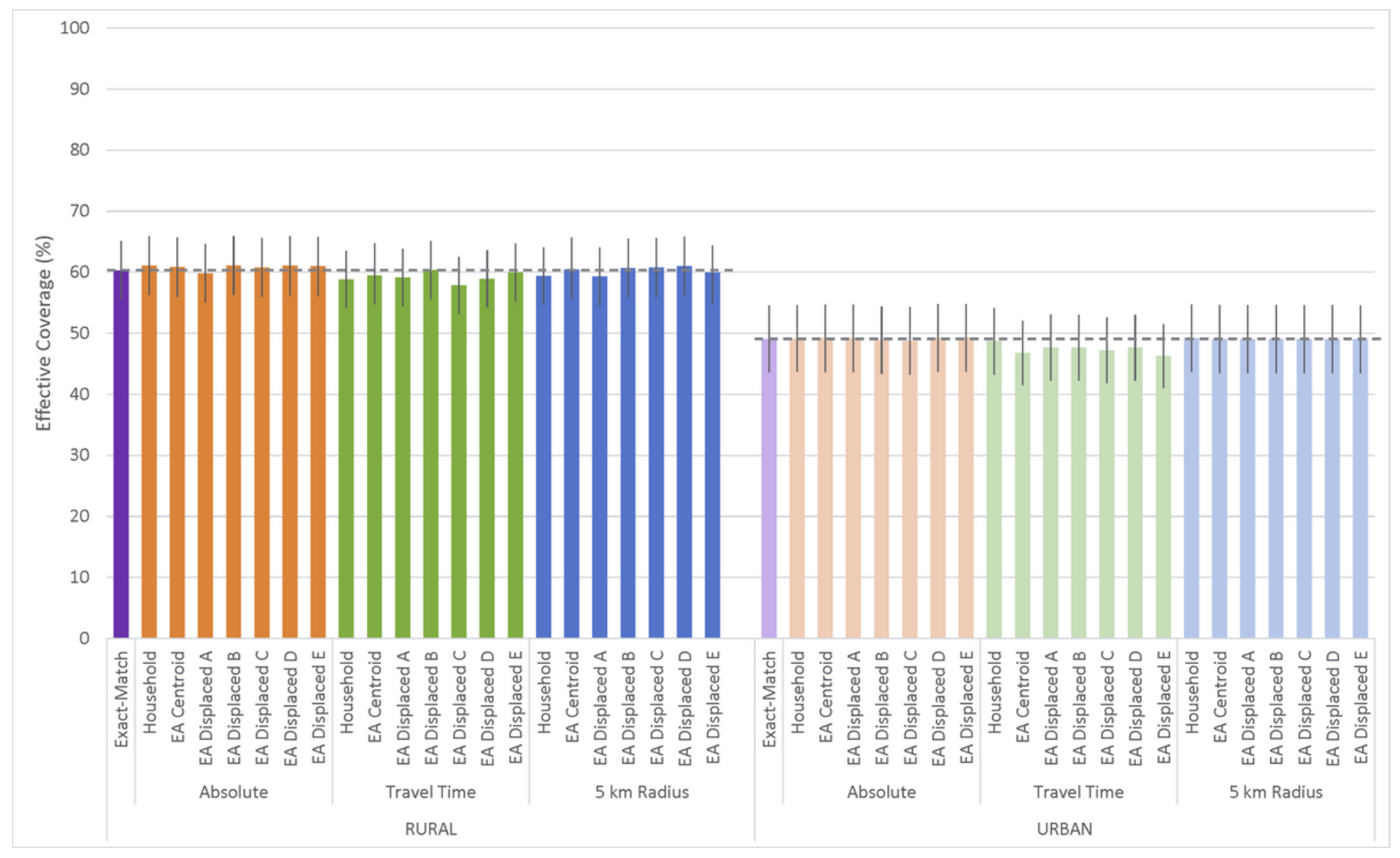

\section{Figure 4}

Effective coverage of management of child illness by linking method and child location 